\title{
Fragile Glasses Associated with a Dramatic Drop of Entropy under Supercooling
}

\author{
Chun-Shing Lee $\odot,{ }^{1}$ Matteo Lulli®,${ }^{1,2}$ Ling-Han Zhang $\odot{ }^{3}$ Hai-Yao Deng $\odot,{ }^{4}$ and Chi-Hang Lam $\odot^{1, *}$ \\ ${ }^{1}$ Department of Applied Physics, Hong Kong Polytechnic University, Hong Kong, China \\ ${ }^{2}$ Department of Mechanics and Aerospace Engineering, Southern University of Science and Technology, \\ Shenzhen, Guangdong 518055, China \\ ${ }^{3}$ Department of Physics, Carnegie Mellon University, Pittsburgh, Pennsylvania 15213, USA \\ ${ }^{4}$ School of Physics and Astronomy, Cardiff University, 5 The Parade, Cardiff CF24 3AA, Wales, United Kingdom
}

(Received 29 August 2019; accepted 23 November 2020; published 29 December 2020)

\begin{abstract}
We perform kinetic Monte Carlo simulations of a distinguishable-particle lattice model of structural glasses with random particle interactions. By varying the interaction distribution and the average particle hopping energy barrier, we obtain an extraordinarily wide range of kinetic fragility. A stretching exponent, characterizing structural relaxation, is found to decrease with the kinetic fragility in agreement with experiments. The most fragile glasses are those exhibiting low hopping barriers and, more importantly, dramatic drops of entropies upon cooling toward the glass transition temperatures. The entropy drops reduce possible kinetic pathways and lead to dramatic slowdowns in the dynamics. In addition, the kinetic fragility is shown to correlate with a thermodynamic fragility.
\end{abstract}

DOI: 10.1103/PhysRevLett.125.265703

An important concept in the study of structural glasses [1-3] is the kinetic fragility, often simply called the glass fragility, which has been investigated in great detail for different types of glass formers [4-7]. It describes how rapidly the dynamics slows down when temperature decreases. The dynamics is typically characterized by viscosity, structural relaxation time [8,9], or particle diffusion coefficient [10,11]. Glasses possessing the most dramatic slowdown are classified as fragile, whereas the opposite are referred to as strong. Several models of glasses have been able to reproduce a range of kinetic fragilities [12-16]. A closely related thermodynamic fragility [17] has also been defined and is based on how dramatically the entropy drops as the temperature decreases. Experimental results indicate, in general, a positive correlation between the kinetic and thermodynamic fragilities [17,18]. Yet, a fundamental understanding of the fragilities and their relationship is still lacking.

In this Letter, we study the kinetic and thermodynamic fragilities using a recently proposed distinguishable-particle lattice model (DPLM) of structural glasses [19]. Lattice models are instrumental in statistical physics. Celebrated examples include the Ising model for magnetism and the Edwards-Anderson model for spin glasses [20]. By bridging between analytic theory and more realistic models, they play pivotal roles in the solution and intuitive understanding of the systems concerned. The DPLM aims at this bridging task for the study of structural glass. It possesses exactly solvable equilibrium statistics [19] and is promising for analytical treatment [21,22]. In support of its validity as a model of glass, DPLM has successfully reproduced typical glassy behaviors [19], a remarkable phenomenon known as
Kovacs' expansion gap paradox [23], as well as Kovacs' effect for the aging of glasses [24]. It captures in simpler and more tractable form the relevant physics seen in molecular dynamics (MD) and other realistic models, which in turn are more detailed approximate models of glasses. This should be a worthwhile approach considering that direct analytical treatment of MD or experimental systems in finite dimensions has proved exceedingly challenging and controversial [3].

Here, we show that both the kinetic and thermodynamic fragilities of the DPLM can be varied over wide ranges of values via the fine-tuning of its kinetic and thermodynamic properties. Modeled glasses with higher kinetic fragilities in general exhibit smaller stretching exponents as well as higher thermodynamic fragilities, in good qualitative agreement with experiments. The fundamental mechanisms behind the fragility variations in this model are intuitively understandable, and are likely applicable also to realistic glasses.

We adopt the DPLM proposed in Ref. [19], with minor differences explained in Sec. I in Supplemental Material (SI) [25]. It is defined on a 2D square lattice of size $L^{2}$ with $L=100$ and unit lattice constant following periodic boundary conditions. There are $N$ distinguishable particles on the lattice labeled from 1 to $N$. Each lattice site $i$ can be occupied by at most one of the particles with a particle index $s_{i}=1,2, \ldots, N$. For unoccupied sites, i.e., sites occupied by voids, $s_{i}=0$. A void density of $\phi_{v}=0.01$ is considered. A particle configuration is specified by the set of particle indices $\left\{s_{i}\right\}$ over all sites. The total energy is

$$
E=\sum_{\langle i, j\rangle^{\prime}} V_{s_{i} s_{j}},
$$


where the sum is restricted to nearest neighbor sites $i$ and $j$ occupied by particles. The interaction $V_{k l}$ for each pair of adjacent particles $k$ and $l$ is sampled before the start of the simulation from the pair-interaction distribution $g\left(V_{k l}\right)$ and fixed subsequently. The particle index $s_{i}$ is time dependent since the site $i$ will be visited by different particles as the system evolves. Thus, $V_{s_{i} s_{j}}$ in Eq. (1) is time dependent, although any $V_{k l}$ for any given particles $k$ and $l$ is quenched. Dimensionless units will be adopted.

Particle distinguishability and particle-dependent interactions are directly justifiable for polydispersive or polymer systems. For identical-particle systems, it instead accounts effectively for the generally different frustration states experienced by the particles. It also models high-entropy alloys [35] in the limit of a large number of atomic species. Being a lattice model, particle vibrations are not explicitly accounted for. A particle configuration more precisely models an inherent state of a realistic system [22].

A main feature of our work is the random sampling for each $V_{k l} \in\left[V_{0}, V_{1}\right] \equiv[-0.5,0.5]$ from a bicomponent distribution consisting of a uniform and a delta function representing, respectively, unexcited and excited states given by

$$
g(V)=\frac{G_{0}}{\Delta V}+\left(1-G_{0}\right) \delta\left(V-V_{1}\right)
$$

where $\Delta V=V_{1}-V_{0}=1$ and $\delta$ denotes the Dirac delta function. Here, $G_{0} \in[0,1]$ is our main thermodynamic parameter controlling the fragilities. It equals the probabilistic weight of the uniform unexcited component of the distribution and also the probability density $g\left(V_{0}\right)$ at the ground state energy $V_{0}$. For $G_{0}=1$, Eq. (2) reduces to the uniform distribution adopted in Ref. [19], which leads to a strong glass. Alternatively, for $G_{0}=0$, all interactions are at the excited energy state $V_{1}$ and the model reduces to a simple identical-particle lattice gas with a uniform particle interaction.

We assume a void-induced dynamics, which has been directly observed in recent experiments on glassy colloidal systems [36]. Using the Metropolis algorithm, each particle can hop to an unoccupied nearest neighbor site at temperature $T$ at a rate

$$
w= \begin{cases}w_{0} \exp \left[-\left(E_{0}+\Delta E\right) / k_{B} T\right] & \text { for } \Delta E>0, \\ w_{0} \exp \left(-E_{0} / k_{B} T\right) & \text { for } \Delta E \leq 0,\end{cases}
$$

where $\Delta E$ is the change in the system energy $E$ given by Eq. (1) due to the hop and $k_{B}=1$ is the Boltzmann constant. We put $w_{0}=10^{6}$. The hopping energy barrier offset $E_{0} \geq 0$ is our main kinetic model parameter for controlling the fragilities. Our algorithm satisfies detailed balance.

Kinetic Monte Carlo simulations have been performed on the DPLM, starting from directly constructed initial

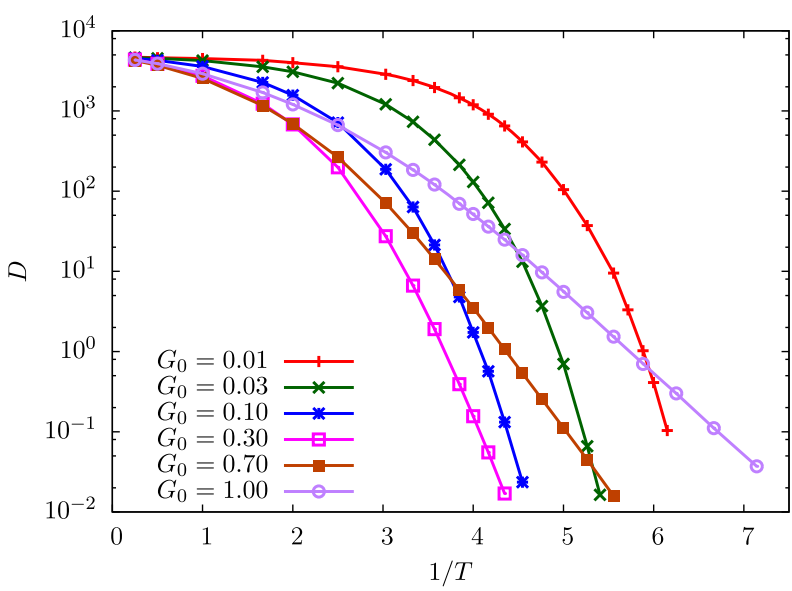

FIG. 1. Arrhenius plot of $D$ for various $G_{0}$ at $E_{0}=0$. The system with a lower $G_{0}$ is more super-Arrhenius.

equilibrium configurations [19]. We report here our main results while further details are given in Sec. II in SI [25]. The particle mean squared displacement defined as $\mathrm{MSD}=\left\langle\left|\mathbf{r}_{l}(t)-\mathbf{r}_{l}(0)\right|^{2}\right\rangle$ is calculated, where $\mathbf{r}_{l}(t)$ denotes the position of particle $l$ at time $t$. The particle diffusion coefficient $D$ is computed according to $D=(1 / 2 d)(\mathrm{MSD} / t)$, where $d=2$ is the dimension of the system, at sufficiently large values of $t$ in the diffusion regime.

The Arrhenius plot in Fig. 1 shows $D$ against $1 / T$ for $E_{0}=0$ and various $G_{0}$. We observe that $\log D$ decreases with $1 / T$ faster than linearly, demonstrating a superArrhenius slowdown. The dependence of $D$ on $G_{0}$ for any given $T$ is nonmonotonic. Yet, the super-Arrhenius behavior strengthens monotonically as $G_{0}$ decreases. This can be clearly seen in a kinetic Angell plot in Fig. 2, which plots $D^{-1}$ against $T_{g} / T$ for $E_{0}=0$ (solid lines) using the data from Fig. 1. We have defined the glass transition temperature $T_{g}$ as $T$ at which $D=D_{r} \equiv 10^{-1}$, where the reference diffusion coefficient $D_{r}$ is about the lowest value we can simulate. We observe that $D$ now varies monotonically with $G_{0}$ for any given $T_{g} / T$. More importantly, the super-Arrhenius property clearly strengthens monotonically as $G_{0}$ decreases. Related kinetic Angell plot of structural relaxation time extracted from self-intermediate scattering function shows closely analogous trends (see Sec. II in SI [25]).

Figure 2 also shows $D^{-1}$ for $E_{0}=1$ (dotted lines). Results are simply obtained from values of $D$ for $E_{0}=0$ after rescaling time by a factor $\exp \left(E_{0} / k_{B} T\right)$, noting that $T_{g}$ has to be recalculated since $D_{r}$ is not rescaled. We observe that a smaller $E_{0}$ strengthens the super-Arrhenius property at any given $G_{0}$. The results in Fig. 2 capture many qualitative features in experimental findings [5-7].

The kinetic fragility $m_{k}$ describes the super-Arrhenius property quantitatively and is defined by $m_{k}=$ $\partial \log D^{-1} /\left.\partial\left(T_{g} / T\right)\right|_{T=T_{g}}$. We obtain a wide range of values 


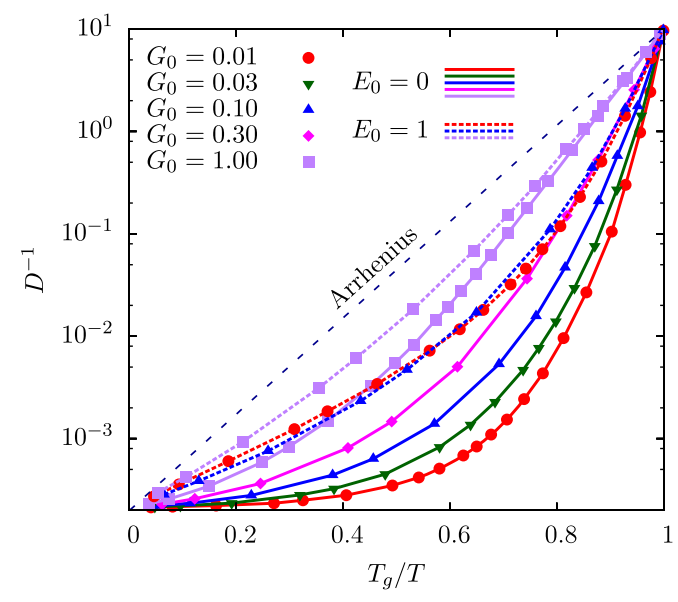

FIG. 2. Kinetic Angell plot of $D^{-1}$ against $T_{g} / T$ for various $G_{0}$ and $E_{0}$, where $T_{g}$ for each curve is defined at $D_{r}=10^{-1}$. A low $G_{0}$ gives a fragile system. For a given $G_{0}$, increasing $E_{0}$ makes the system stronger.

of $m_{k}$ from 6.76 to 26.35 . These values are in general smaller than experimental ones typically in the range from 25 to 150 [37], but this is only due to a rather small $D_{r}$ adopted for defining $T_{g}$. An extrapolation to $D_{r}=10^{-14}$ is performed so that nearly 18 orders of magnitude of $D$ are considered, similar to analyses of structural relaxation time and viscosity in experiments [8,9]. Then, $m_{k}$ ranges from 21.4 for large $G_{0}$ and $E_{0}$ and 120 for $G_{0}=0.01$ and $E_{0}=0$, consistent with the experimental range (see Sec. III in SI [25]).

To further establish the physical relevance of the DPLM, we proceed to show that relaxation and thermodynamic properties of the strong and fragile glasses from this model are consistent with experiments. First, structural relaxation is studied by measuring the self-intermediate scattering function

$$
F_{s}(\mathbf{q}, t)=\left\langle e^{i \mathbf{q} \cdot\left(\mathbf{r}_{l}(t)-\mathbf{r}_{l}(0)\right)}\right\rangle,
$$

where $q=(2 \pi / L) q^{\prime}$ with $q^{\prime}=10$. The results are nicely fitted by the stretched exponential function $A \exp \left[-(t / \tau)^{\beta}\right]$ for $t \gtrsim \tau$, where $\beta, \tau$, and $A$ are, respectively, the stretching exponent, the relaxation time, and a constant close to unity. Figure 3 plots $m_{k}$ against $\beta$ at $T_{g}$ for various $G_{0}$ and $E_{0}$. It shows that $m_{k}$ tends to decrease approximately linearly with $\beta$, in agreement with a trend observed previously in experiments [37]. In addition, the obtained range 0.37 to 0.81 of $\beta$ is comparable to that from experiments. Results on $\beta$ are not significantly affected by using smaller values of $D_{r}$, especially for the fragile glasses since $T_{g}$ only changes slightly.

Second, we study the thermodynamic properties of our model by calculating an entropy-based thermodynamic fragility. The equilibrium statistics including the partition function $Z$ of the DPLM are exactly known [19].

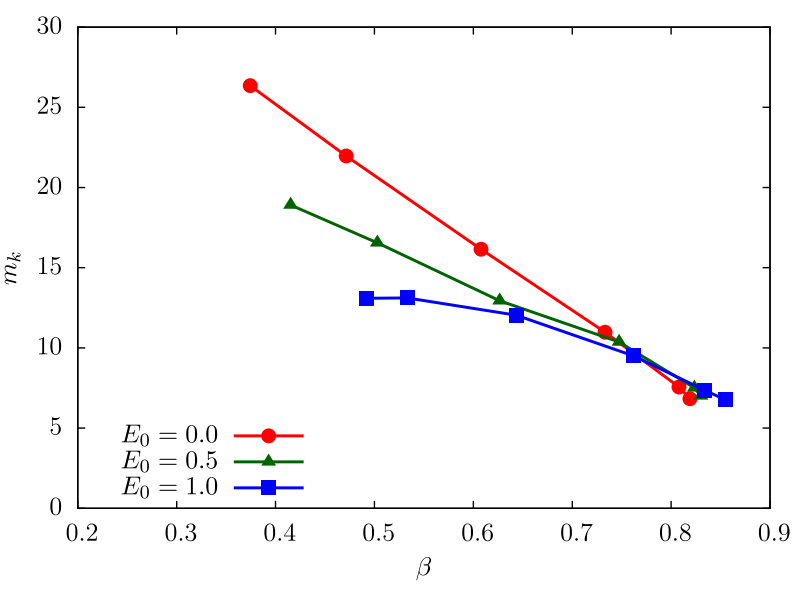

FIG. 3. Relationship between $m_{k}$ and $\beta$ at $E_{0}=0,0.5,1$ with $G_{0}=0.01,0.03,0.1,0.3,0.7,1$ (from left to right).

The entropy per particle $s(T)$ is computed accordingly. We further define an excess entropy per particle $s^{\mathrm{ex}}(T)=$ $s(T)-s^{\mathrm{LG}}$ over the entropy $s^{\mathrm{LG}}$ of a simple lattice gas [38]. [See Eq. (S23).] The inset of Fig. 4 shows a thermodynamic Angell plot of $-s^{\mathrm{ex}}(T) /\left|s^{\mathrm{ex}}\left(T_{g}\right)\right|$ against $T_{g} / T$ for $E_{0}=0$ and different $G_{0}$. The results resemble those of closely related thermodynamic Angell plots from experiments [39] as well as the kinetic Angell plot in Fig. 2. An increased $E_{0}$ alters the curvature only slightly for all values of $G_{0}$. In general, a strong glass with $G_{0}=1$ is also thermodynamically strong with a close-to-linear relation, while a fragile glass at $G_{0}=0.01$ shows the most dramatic variations. The trend is in general similar if other forms of thermodynamic Angell plots [39] are considered.

We define a thermodynamic fragility $m_{t}$ as

$$
m_{t}=\left.\frac{\partial\left(-s^{\mathrm{ex}}(T) /\left|s^{\mathrm{ex}}\left(T_{g}\right)\right|\right)}{\partial\left(T_{g} / T\right)}\right|_{T=T_{g}},
$$

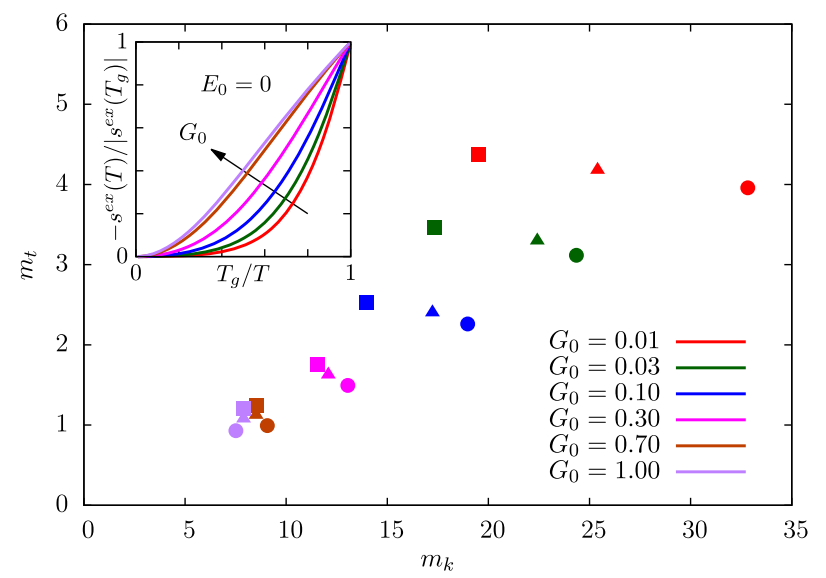

FIG. 4. Plot of $m_{t}$ against $m_{k}$ at $E_{0}=0$ (circle), 0.5 (triangle), and 1 (square) for various $G_{0}$. Inset: Thermodynamic Angell plot of $-s^{\mathrm{ex}}(T) /\left|s^{\mathrm{ex}}\left(T_{g}\right)\right|$ at $E_{0}=0$. 
which is analogous to the kinetic counterpart $m_{k}$. Figure 4 shows the kinetic fragility $m_{t}$ against the thermodynamic fragility $m_{k}$ for various $G_{0}$ and $E_{0}$, displaying a clear tendency of a positive correlation, i.e., $m_{t} \sim m_{k}$. The correlation is consistent with the general trend observed in experiments based on related definitions [39] and is mainly caused by the similar dependencies of $m_{k}$ and $m_{t}$ on $G_{0}$.

We have studied glass fragility using the DPLM for various values of model parameters $G_{0}$ and $E_{0}$. The most fragile glass is obtained at small $G_{0}$ and $E_{0}=0$. Extrapolating our simulation results toward $G_{0} \rightarrow 0$, the kinetic fragility $m_{k}$ appears to rise unboundedly (see Fig. S12). The DPLM may hence model in-principle arbitrarily fragile glasses. Simulations at very small $G_{0}$ are, however, prohibitively intensive due to increased finite-size effects. At $G_{0}=0$, the model reduces to a simple lattice gas, which is not glassy. A high $m_{k}$ thus requires a small but nonvanishing probability of low-energy particle pairings.

We argue that $G_{0}$ is the main material parameter which captures the relevant particle interaction characteristics and determines the fragilities $m_{t}$ and $m_{k}$ in glasses. The value of $G_{0}$ in a glass depends on the detailed molecular interactions and is strongly affected, for example, by the geometries of any tightly bounded groups of atoms. A fragile glass obtained at a small $G_{0}$ can be intuitively understood as follows. At high $T$, all particle configurations are possible, leading to a high entropy $s(T)$ independent of $G_{0}$. Most interactions take the excited states with energy $V_{1}$ due to their high probabilistic weight $1-G_{0}$ [see Eq. (2)]. Particle pairings with unexcited energies close to $V_{0}$ are in contrast rare due to the small probabilistic weight $G_{0}$. As $T$ decreases, the lower energies render them energetically favorable and increasingly dominant. The entropy $s(T)$ thus drops dramatically and becomes small at low $T$, accounting for a high $m_{t}$.

We further suggest that this high $m_{t}$ is closely correlated to a high $m_{k}$. This is because the system dynamics at low $T$ amounts to sampling various energetically favorable configurations. The rarity of these configurations as indicated by the low entropy implies highly constrained kinetic pathways of particle motions. This leads to a sharp drop in $D$ as described by a large $m_{k}$. A possible characteristic of constrained kinetics is repetitive particle motions. Our picture is thus supported by a sharp increase in a particle return probability but a mild drop of the particle hopping rate as $T$ decreases as explained in Sec. II in SI [25]. The thermodynamic parameter $G_{0}$ therefore strongly impacts the system thermodynamics and hence also the kinetics. In contrast, the kinetic parameter $E_{0}$ is of lesser importance to the fragility properties. By controlling the hopping barrier, it clearly has a strong and direct impact on $m_{k}$. However, it plays no role in the equilibrium statistics and in particular in the system entropy [see Eq. (S23)]. It has a tiny impact on $m_{t}$ only by influencing the value of $T_{g}$ at which $m_{t}$ is evaluated. Not accounting for the correlation between $m_{k}$ and $m_{t}$ observed in experiments, we expect $E_{0}$ to play a smaller role in the variation of $m_{k}$ among various glasses.

The particle interaction distribution $g(V)$ has been taken with a bicomponent form consisting of a low-energy uniform distribution and a high-energy delta function for simplicity. The delta function represents excited particle interactions more relevant at higher $T$ and replacing it by some narrow Gaussian leads to similar simulation results. The uniform distribution is the simplest continuous distribution with a lower bound $V_{0}$, corresponding to the energy minimum present in typical pair potentials such as the Lennard-Jones potential. The continuous form of $g(V)$ around $V_{0}$ is expected to lead to glassy behaviors even at a very low $T$, as the model reduces to one with a single uniform distribution studied in Ref. [19].

The DPLM with a bicomponent $g(V)$ is closely related to a bond excitation model proposed by Moynihan and Angell [40], in which particle bonds can assume either an unexcited or excited state (see Sec. VI in SI [25]). At low $T$, the realized interactions $V_{s_{i} s_{j}}$ from the uniform unexcited component have a small energy spread of about $k_{B} T$ around $V_{0}+k_{B} T$. Neglecting this energy spread, the ratio of the degeneracy of the excited states to that of the unexcited states is about $\left(1-G_{0}\right) / G_{0}$, leading to an entropy difference

$$
\Delta S^{0} \simeq k_{B} \ln \left[\left(1-G_{0}\right) / G_{0}\right] .
$$

Considering $G_{0}=0.01$ corresponding to fragile glasses, we get $\Delta S^{0} \simeq 4.60 k_{B}$. A more accurate calculation using Eq. (S45) gives a similar value of $\Delta S^{0} \simeq 5.42 k_{B}$. Reverting to physical units with $k_{B}=8.315 \mathrm{~J} / \mathrm{mol} \mathrm{K}$, it gives $\Delta S^{0} \simeq 45.1 \mathrm{~J} / \mathrm{K}$ per mole of excitable states. This value matches that of $\Delta S^{0}$, for example, for toluene in Ref. [40], which has a high $m_{k}=103$. In addition, $\Delta H^{0} \simeq 1-k_{B} T_{g}$ is the energy difference between the excited and unexcited states in our model. At $T_{g} \simeq 0.163, \Delta H^{0} / k_{B} T_{g} \simeq$ $(1-0.163) / 0.163 \simeq 5.15$ for $G_{0}=0.01$. It compares well with the value 6.95 for toluene in Ref. [40].

The quantitative consistency demonstrated above means that the bond excitation model provides a simplified theoretical description for the thermodynamic properties of the DPLM with the bicomponent $g(V)$. Moreover, the success of the bond excitation model in describing the entropy of fragile glasses in Ref. [40] justifies the bicomponent form of $g(V)$ used in this work. From Eq. (6), a fragile glass characterized by a small $G_{0}$ possesses a large $\Delta S^{0}$. These material parameters depend on the detailed molecular interactions. For molecular or polymer glasses which are often fragile, their values may reflect that the geometrically complex molecules fit well with each other to form very stable bonds only at a rare set of orientations and conformations. In contrast, strong glasses including network glasses may consist of simpler structures such as 
tetrahedrons. A simple random spread of the interactions due to frustration can then account for $G_{0} \simeq 1$ and a small $\Delta S^{0}$.

We have found that the thermodynamic parameter $G_{0}$ has the strongest impacts on both $m_{k}$ and $m_{t}$. In contrast, the kinetic parameter $E_{0}$ also plays a significant role for $m_{k}$ but not so much for $m_{t}$. Further simulations show that the void density $\phi_{v}$ has rather small effects on both $m_{k}$ and $m_{t}$, as long as $\phi_{v} \ll 1$ which ensures the glassy state. One can also consider model variations such as a different $g(V)$. Since glass properties depend on multiple model parameters, the relations discussed here between $m_{k}, m_{t}$, and $\beta$ are only general trends assuming small variations in other parameters. Exceptions are thus possible in more general settings. From another point of view, the value of $m_{k}$ does not uniquely determine the precise geometry of the whole curve in the Angell plot in Fig. 2 when multiple material parameters are taken into account. These are fully consistent with experimental observations [5].

To sum up, we have studied fragility properties of glasses using kinetic Monte Carlo simulations and analytic calculations based on the DPLM. A wide range of values of kinetic fragility is reproduced, indicating the possibility of arbitrarily fragile glasses limited only by computational resources. The kinetic fragility is mainly controlled via a thermodynamic parameter $G_{0}$, dictating the probability distribution of particle pair interactions. The most fragile glass is obtained at small $G_{0}$ corresponding to the case that pair interactions can take low-energy states with a small but nonvanishing probability, i.e., low-entropy unexcited states. These configurations physically represent rare pairings between particles with exceptionally stable arrangements. As the temperature decreases, particle configurations are increasingly constrained to these low-energy pairings. This causes a dramatic drop in the entropy associated with a dramatic slowdown in the dynamics, resulting, respectively, in high thermodynamic and kinetic fragilities. Our model, upon variations in $G_{0}$, exhibits correlations between kinetic fragility, thermodynamic fragility, and a relaxation stretching exponent, in qualitative agreement with general trends observed in experiments. The kinetic fragility is also affected by a kinetic model parameter $E_{0}$. A fragile glass is obtained at small $E_{0}$ corresponding to particle hopping activation barriers with an average which is small compared to their fluctuations.

We thank the support of Hong Kong General Research Fund (GRF) (Grant No. 15330516), Hong Kong PolyU (Grant No. 1-ZVGH), and National Natural Science Foundation of China (Grant No. 11974297).

*C.H.Lam@polyu.edu.hk

[1] L. Berthier and G. Biroli, Theoretical perspective on the glass transition and amorphous materials, Rev. Mod. Phys. 83, 587 (2011).
[2] J. P. Garrahan, P. Sollich, and C. Toninelli, Kinetically constrained models, in Dynamical Heterogeneities in Glasses, Colloids and Granular Media, edited by L. Berthier, G. Biroli, J.-P. Bouchaud, L. Cipelletti, and W. van Saarloosand (Oxford University Press, Oxford, 2011).

[3] F. H. Stillinger and P. G. Debenedetti, Glass transition thermodynamics and kinetics, Annu. Rev. Condens. Matter Phys. 4, 263 (2013).

[4] R. Bohmer, K. L. Ngai, C. A. Angell, and D. J. Plazek, Nonexponential relaxations in strong and fragile glass formers, J. Chem. Phys. 99, 4201 (1993).

[5] C. A. Angell, Formation of glasses from liquids and biopolymers, Science 267, 1924 (1995).

[6] L.-M. Wang, C. Austen Angell, and R. Richert, Fragility and thermodynamics in nonpolymeric glass-forming liquids, J. Chem. Phys. 125, 074505 (2006).

[7] D. Giordano and D. B. Dingwell, The kinetic fragility of natural silicate melts, J. Phys. Condens. Matter 15, S945 (2003).

[8] C. A. Angell, Relaxation in liquids, polymers and plastic crystals-Strong/fragile patterns and problems, J. NonCryst. Solids 131-133, 13 (1991).

[9] C. Alba, L. E. Busse, D. J. List, and C. A. Angell, Thermodynamic aspects of the vitrification of toluene, and xylene isomers, and the fragility of liquid hydrocarbons, J. Chem. Phys. 92, 617 (1990).

[10] F. Fujara, B. Geil, H. Sillescu, and G. Fleischer, Translational and rotational diffusion in supercooled orthoterphenyl close to the glass transition, Z. Phys. B 88, 195 (1992).

[11] D. Coslovich and G. Pastore, Understanding fragility in supercooled lennard-jones mixtures. I. Locally preferred structures, J. Chem. Phys. 127, 124504 (2007).

[12] J. P. Garrahan and D. Chandler, Geometrical Explanation and Scaling of Dynamical Heterogeneities in Glass Forming Systems, Phys. Rev. Lett. 89, 035704 (2002).

[13] F. Sausset, G. Tarjus, and P. Viot, Tuning the Fragility of a Glass-Forming Liquid by Curving Space, Phys. Rev. Lett. 101, 155701 (2008).

[14] A. Parmar and S. Sastry, Kinetic and thermodynamic fragilities of square well fluids with tunable barriers to bond breaking, J. Phys. Chem. B 119, 11243 (2015).

[15] O. Misaki, K. Kang, and M. Kunimasa, Tuning pairwise potential can control the fragility of glass-forming liquids: From a tetrahedral network to isotropic soft sphere models, J. Stat. Mech. (2016) 074002.

[16] S. Ciarella, R. A. Biezemans, and L. Janssen, Understanding, predicting, and tuning the fragility of vitrimeric polymers, Proc. Natl. Acad. Sci. U.S.A. 116, 25013 (2019).

[17] C. A. Angell and K. Ueno, Soft is strong, Nature (London) 462, 45 (2009).

[18] D. F. Giulia and B. Livio, Thermodynamic and dynamic fragility in metallic glass-formers, Acta Mater. 61, 2260 (2013).

[19] L.-H. Zhang and C.-H. Lam, Emergent facilitation behavior in a distinguishable-particle lattice model of glass, Phys. Rev. B 95, 184202 (2017).

[20] S. F. Edwards and P. W. Anderson, Theory of spin glasses, J. Phys. F 5, 965 (1975). 
[21] C.-H. Lam, Local random configuration-tree theory for string repetition and facilitated dynamics of glass, J. Stat. Mech. (2018) 023301.

[22] H.-Y. Deng, C.-S. Lee, M. Lulli, L.-H. Zhang, and C.-H. Lam, Configuration-tree theoretical calculation of the meansquared displacement of particles in glass formers, J. Stat. Mech. (2019) 094014.

[23] M. Lulli, C. S. Lee, H. Y. Deng, C. T. Yip, and C. H. Lam, Spatial Heterogeneities in Structural Temperature Cause Kovacs' Expansion Gap Paradox in Aging of Glasses, Phys. Rev. Lett. 124, 095501 (2020).

[24] M. Lulli, L.-H. Zhang, C.-S. Lee, H.-Y. Deng, and C.-H. Lam, Kovacs effect studied using the distinguishable particles lattice model of glass, arXiv:1910.10374.

[25] See Supplemental Material at http://link.aps.org/ supplemental/10.1103/PhysRevLett.125.265703 for the details of the model and other supplemental results, which includes Refs. [26-34].

[26] T. Damart and D. Rodney, Atomistic study of two-level systems in amorphous silica, Phys. Rev. B 97, 014201 (2018).

[27] W. Kob and H. C. Andersen, Kinetic lattice-gas model of cage effects in high-density liquids and a test of modecoupling theory of the ideal-glass transition, Phys. Rev. E 48, 4364 (1993).

[28] C. Y. Liao and S-H Chen, Dynamics of inherent structure in supercooled liquids near kinetic glass transition, Phys. Rev. E 64, 031202 (2001).

[29] C.-H. Lam, Repetition and pair-interaction of string-like hopping motions in glassy polymers, J. Chem. Phys. 146, 244906 (2017).
[30] C.-H. Lam, Deeper penetration of surface effects on particle mobility than on hopping rate in glassy polymer films, J. Chem. Phys. 149, 164909 (2018).

[31] A. Nußbaumer, J. Zierenberg, E. Bittner, and W. Janke, Numerical test of finite-size scaling predictions for the droplet condensation-evaporation transition, J. Phys. 759, 012009 (2016).

[32] M. Toda, R. Kubo, and N. Saito, Statistical Physics I: Equilibrium Statistical Mechanics (Springer, Heidelberg, 1991).

[33] L. Onsager, Statistical hydrodynamics, Il Nuovo Cimento (1943-1954) 6, 279 (1949).

[34] C. N. Yang, The spontaneous magnetization of a twodimensional Ising model, Phys. Rev. 85, 808 (1952).

[35] J.-W. Yeh, Alloy design strategies and future trends in highentropy alloys, JOM 65, 1759 (2013).

[36] C.-T. Yip, M. Isobe, C.-H. Chan, S. Ren, K.-P. Wong, Q. Huo, C.-S. Lee, Y.-H. Tsang, Y. Han, and C.-H. Lam, Direct Evidence of Void-Induced Structural Relaxations in Colloidal Glass Formers, Phys. Rev. Lett. 125, 258001 (2020).

[37] M. Matthieu, Relaxation and physical aging in network glasses: A review, Rep. Prog. Phys. 79, 066504 (2016).

[38] J. C. Dyre, Perspective: Excess-entropy scaling, J. Chem. Phys. 149, 210901 (2018).

[39] L. M. Martinez and C. A. Angell, A thermodynamic connection to the fragility of glass-forming liquids, Nature (London) 410, 663 (2001).

[40] C. T. Moynihan and C. A. Angell, Bond lattice or excitation model analysis of the configurational entropy of molecular liquids, J. Non-Cryst. Solids 274, 131 (2000). 